\title{
A Fast Method for Monitoring Driver Fatigue Using Monocular Camera
}

\author{
Hongbiao Ma, Zehong Yang, Yixu Song, Peifa Jia \\ State Key Laboratory on Intelligent Technology and Systems, Tsinghua \\ National Laboratory for Information Science and Technology, Department of \\ Computer Science and Technology, Tsinghua University, Beijing 100084, China \\ ma-hb06@mails.tsinghua.edu.cn
}

\begin{abstract}
Driver fatigue is an important factor in a large number of accidents. In this paper, a real time vision-based method is proposed to monitor driver fatigue. Firstly, the face is located by a Haar-like feature based object detection algorithm, Secondly, eye is detected and eyelid distance is computed. By analyzing the changes of the distance of eyelid along with time, we can detect driver's fatigue and issue a warning. The main advantage of this system is its extreme high speed and very simple equipment. This system runs at about 15 frames per second in video with 640*480resolution.
\end{abstract}

Keywords: driver fatigue monitoring, computer vision, monocular camera, face detection

\section{INTRODUCTION}

Fatigue has been widely accepted as a main factor causing vehicle accidents. According to the National Highway Traffic Safety Administration (NHTSA) [1] estimates, 100000 police-reported crashes are directly caused by driver fatigue each year, which results in an estimated 1550 deaths, 71000 injuries, and $\$ 12.5$ billion losses. In 2002, the National Sleep Foundation (NSF) reported that $51 \%$ adult drivers had driven a vehicle while feeling drowsy and $17 \%$ had actually fallen asleep.
The Federal Motor Carrier Safety Administration (FMCSA), the trucking industry, highway safety advocates, and transportation researchers have all identified driver drowsiness as a high priority commercial vehicle safety issue. Drowsiness affects mental alertness, decreasing an individual's ability to operate a vehicle safely and increasing the risk of human error that could lead to fatalities and injuries. Furthermore, it has been shown to slow reaction time, decreases awareness, and impairs judgment.

Developing technologies for monitoring the driver fatigue is essential to prevent vehicle accident. People in fatigue exhibit certain visual behaviors that are easily observable from changes in facial features like the eyes, head and face. Visual behaviors that typically reflect a person's level of fatigue include eyelid movement, head movement, gaze, and facial expression [3].

In recent years, there have been many studies [3-7] focusing on this topic using visual behaviors. However, some of these studies use very complicated hardware, costing too much money and energy; some use simple hardware but run at a relatively low speed. In this paper, we choose the eyelid movement as the measurement parameter and propose a realtime system for monitoring driver fatigue using just one camera.

This paper is organized as follows: in section 2, a haar algorithm is used to locate the face and detect the eye in the interested region; in section 3 , an automatic

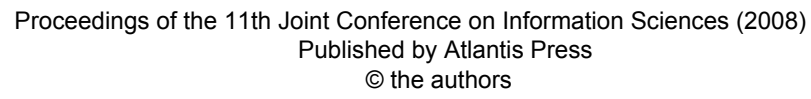


threshold algorithm is applied to get the binary eye contour image, then moving average eyelid distance is computed to decide whether driver is fatigue or not; in section 4, we make a conclusion and point out the future direction.

\section{FACE DETECTION AND EYE DETECTION}

Face detection and eye detection are all accomplished by the haar algorithm [8] proposed by Viola and Jones. We find that, due to the complex background, it is not a good choice to locate or detect the right eye in the original image, for it will take much more time on searching the whole window with poor results. So we firstly find the location of face, and reduce the range in which we will detect the right eye. Doing this can improve the tracking speed and correct rate, reduce the affect of the complex background. Besides, we propose a very simple but powerful method to reduce the computing complexity.

\subsection{Face detection}

Prior to the eye localization, a robust face detector described in [8] is applied to extract face images from video frames (inside the red rectangle, see figure1.a). This original detector runs less than 10 frames per second when used in video with $640 * 480$ resolutions, which is not an acceptable result we want. We will optimize it to reduce the time it costs in detecting one frame. Here we propose a very simple but powerful method to reduce the computational complexity on detecting the face.

Since it is developed for fatigue monitoring in vehicle environment, we assume that there is only one person in video. So we can make some improvement by reducing the searching region from the whole image to the nearby region around the previous detected face. There are 2 parameters which will greatly influence the computing time in the haar-based object detection algorithm, one is the Region of Interest(ROI) of the image (denoted as FACE_ROI) in which range we will detect the face, the other is the minimum searching window (denoted as MIN_WND). In the beginning, we set the MIN_WND as a very small square and let the FACE_ROI cover the whole image. Once we have detected the face, since most time driver's face will not move rapidly and the size of face will not vary sharply, we can set the FACE_ROI as a square with side length $r^{*} 6 / 5$ concentric with previous detected face, and set the MIN_WND as a square with side length $r^{*} 9 / 10$ ( $r$ is the side length of previous detected face, the factors are empirically set for best result). If no face is detected, the detector will expend FACE_ROI and reduce MIN_WND until face is found or boundary is reached. Using this simple method, we can reduce the time of detecting face in every frame from about $130 \mathrm{~ms}$ to about $40 \mathrm{~ms}$.

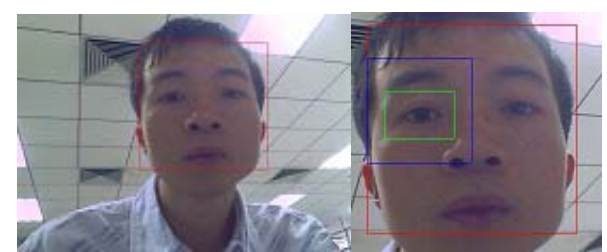

Figure.1: (a) Example of face localization, (b) Right eye extraction: big, middle, small rectangles represent regions of face, eye ROI, eye, respectively

\subsection{Eye detection}

As mentioned at the beginning of section 2 , it is difficult and slow to detect the right eye in the original image, we choose to extract the right eye ROI(region of interest) firstly (inside the middle rectangle, see figure1.b). Here we set the width and height of right eye region as $1 / 2$ times of the width and height of the face. Let the

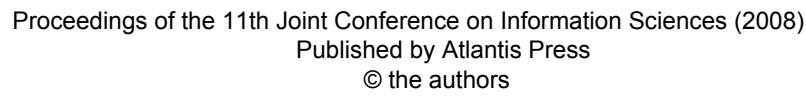


rectangle of face be $[0,0, \mathrm{w}, \mathrm{h}]$, we set the rectangle of right eye ROI as $[0, \mathrm{~h} / 6, \mathrm{w} / 2, \mathrm{~h} / 2]$ (see the middle rectangle in Fig1.b).

After extract the right eye ROI, we can detect the right eye (inside the small rectangle, see Figure1.b)using the haar algorithm in the interest region. Experiment results show that this method can greatly reduce the time on searching the eye. Besides, the rate of detecting wrong eye is decreased.

\section{FATIGUE DETECTION}

There are many cues which can be used for detecting fatigue, like eyelid movement, head movement, gaze, facial expression [3], among which the eyelid movement reflects the state of fatigue most well. Here we propose an automatic threshold method based on histogram to get the eye contour, and compute the distance of eyelid. We find that this method works very well.

\subsection{Automatic threshold}

After we get the eye region image, we change it from color image to gray image (Figure2.a), then use the automatic threshold algorithm to get the eye contour with only eyeball and eyelid. Here we calculate the histogram of eye (Figure.2.b), let $\mathrm{H}$ be the index with max value in the histogram (the $\mathrm{x}$-coordinate with biggest $y$ value), we find that $\mathrm{H}^{*} 2 / 3$ is a good threshold to segment the eye contour (Figure.2.c) from the skin around the eye.

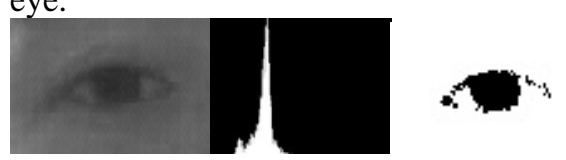

Figure.2: a) gray eye. b) histogram of eye c) binary eye contour with eyeball and eyelid

\subsection{Compute eyelid distance}

Eyelid distance is defined as the distance from upper eyelid to lower eyelid. The eyelid distance is relatively large when driver is clear-minded, and it becomes small when driver feel fatigue. Figure3 shows three difference eye states: open, half open and closed.

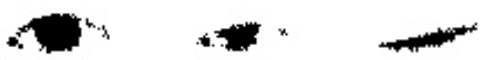

Fig. 3: a) open eye; b) half open eye; c) closed eye

\subsection{Fatigue detection}

Through the above method, we can get the distance of eyelid. By analyzing the state and changing trend of eyelid distance, we can clearly decide whether driver is clear-headed, drowsy or even asleep [Fig 4].

It is assumed that the driver is clearminded at the beginning [Fig4.a] and the distance of the eyelid that time is regarded as the normal value. The eyelid distance is large in most time, with occasional small eyelid denotes eye blinking.

We calculate moving average of eyelid distance in continuous 100 frames. When the moving average is below a threshold (like $60 \%$ of normal value), driver is judged to be fatigue with a warning issued [Fig4.c].
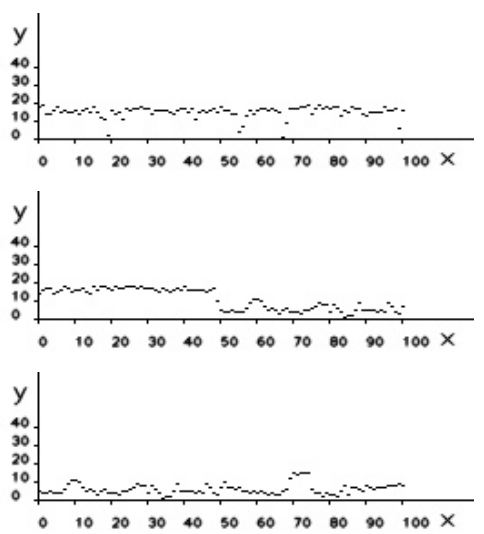
Figure.4: a) clear-minded state, b) driver becomes fatigue, c) driver in totally fatigue. $\mathrm{X}$ axis denotes the number of frame, $\mathrm{Y}$ axis denotes eyelid distance

\section{CONCLUSION}

This paper proposes a real time driver fatigue monitoring method. Haar algorithm is used to locate the face and detect the eye, histogram-based automatic threshold algorithm is used to extract the eye contour, finally we use the moving average of eyelid distance to decide whether driver is fatigue or not.

This algorithm is implemented in $\mathrm{C}++$ with free source OpenCV library. When tested in a PC with Intel Pentium 4 3.0GHz CPU, the overall computing time for one frame is about $65 \mathrm{~ms}$.

However, it should be noted that we concentrate on only eyelid which may encounter difficulty when driver wears glasses. Our future work will try integrate some other cues like head movement, gaze and so on. Moreover, statistical learning method will be applied to better classify the driver's state.

\section{Acknowledge}

This work is supported by The National High Technology Research and Development Program of China.

\section{References}

[1] NHTSA, "Drowsy drivers detection and warning system for commercial vehicle drivers: Field proportional test design, analysis, and progress" National Highway Traffic Safety Administration, Washington, DC.

[2] L.Barr, H.Howarch, S.Popkin, R

J.Carroll. A Review and Evaluation of Emerging Driver Fatigue Detection Measures and Technologies

[3] Q.Ji, Z. Zhu, and P. Lan. "Real-Time Nonintrusive Monitoring and Prediction of Driver Fatigue," IEEE Transactions on Vehicular Technology, Vol. 53, No. 4, July 2004.

[4] X.Fan, B.Yin and Y.Sun. "Yawning Detection for Monitoring Driver Fatigue", Proceeding of the Sixth International Conference on Machine Learning and Cybernetics, Aug 2007

[5] Q.Wang, J.Yang, M,Ren and Y.Zheng. "Driver Fatigue Detection: A Survey", Proceeding os the $6^{\text {th }}$ World Congress on Intelligent Control and Automation, Jun 2006

[6] D.Bao, Z.Yang, Y.Song. "Projection Function for Driver Fatigue Monitoring with Monocular camera”, Proceedings of the ACM symposium on Applied computing, 2007

[7] Wen-Hui Dong, Xiao-Juan Wu, "Driver Fatigue Detection Based on the Distance of Eyelid” , IEEE Int. Workshop VLSI Design \& Video Tech Suzhou, China, May 28-30,2005.

[8] Paul Viola and Michael J. Jones. Rapid Object Detection using a Boosted Cascade of Simple Features. IEEE CVPR, 2001 